\title{
Libro Electrónico: Los sistemas del cuerpo humano, como herramienta pedagógica para la asignatura de ciencias naturales
}

\section{Jacqueline Rojas Chinchilla}

Profesora de Informática Educativa. Graduada de la Carrera de Informática Educativa de la Universidad Estatal a Distancia; jackerch@gmail.com

Recibido: 17 de abril del 2014

\section{RESUMEN}

Este artículo analiza uno de los principales problemas del sistema educativo costarricense, el cuál consiste en utilizar con gran frecuencia recursos tradicionales como el libro impreso y las fotocopias. Se propone el libro electrónico como un recurso no tradicional que motiva a los alumnos y capta su atención.

\section{PALABRAS CLAVE}

Libro electrónico, recursos educativos tradicionales, actitud, conducta del estudiante.
Aceptado: 2 de mayo del 2014

\section{SUMMARY}

This article discusses one of the main problems of the Costa Rican educational system, which is very often used traditional resources like the printed book and photocopies. The e-book is proposed as a nontraditional resource that encourages students and captures their attention.

\section{KEY WORDS}

E-book, traditional educational resources, attitude, student behavior.

\section{INTRODUCCIÓN}

Uno de los principales materiales didácticos utilizados en la educación costarricense es el libro impreso y las fotocopias, Barbier (2005) define el libro impreso como un conjunto de hojas de papel escritas, ya sean impresas, manuscritas o dibujadas, encuadernadas con tapas, debiendo poseer al menos 50 hojas.

El abuso de herramientas tradicionales, como el libro impreso y las fotocopias, puede generar gran apatía y aburrimiento en los estudiantes, por lo poco agradable y retador que pueden ser en algunos casos. Esta situación se presenta en muchas ocasiones en el sistema educativo costarricense, por la falta de recursos didácticos que contribuyan a incrementar el interés del alumno por su aprendizaje, tal es el caso de la Escuela Santa Rosa, Pérez Zeledón, donde hay un uso excesivo de fotocopias del libro impreso como recurso didáctico en la asignatura de Ciencias. El docente de grado extrae de estos libros los contenidos curriculares dirigidos a los alumnos de sexto grado, empleando fotocopias y utilizando la pizarra, la fotocopia es en blanco y negro y solo muestra 
pedazos fragmentados del libro original, al perderse la magia del color con la fotocopia y dejar de existir el texto en su totalidad integrado como un sistema, se pierde en gran medida el asombro y el interés por los temas sometidos a este mecanismo.

Delgado (2009) menciona que el proceso educativo ha sido y seguirá siendo impactado por la introducción acelerada en la sociedad de las tecnologías. Los programas de estudio, desde la primaria hasta la secundaria, deben considerar y potenciar, de parte de los estudiantes, el uso de las tecnologías de la información y comunicación, considerando que el conocimiento se construye por medio de actividades donde los individuos puedan interactuar entre ellos y con su ambiente material.

Recursos tecnológicos como el libro electróni$\mathrm{co}$, permiten hacer del proceso de enseñanzaaprendizaje una vivencia libre e integral, ya que puede ser diseñado o modificado para que se adapte a las necesidades y posibilidades de cada alumno. Es decir, que los alumnos aprendan a su propio nivel, estilo y ritmo.

Se pretende, en síntesis, ofrecer, una herramienta tecnológica dirigida a los estudiantes de la asignatura de Ciencias de sexto grado de la Escuela Santa Rosa para que puedan cumplir satisfactoria, sistemática y en forma amena con los objetivos de aprendizaje propuesto por el docente.

Para su cumplimiento, el proyecto se propuso alcanzar los objetivos que enseguida se exponen.

\section{Objetivo general}

Proponer el diseño de un libro electrónico a partir del análisis de los enfoques pedagógicos y didácticos desarrollados en el proceso de enseñanza-aprendizaje del tema: Los Sistemas del Cuerpo Humano entre el docente y los estudiantes de sexto grado de la Escuela Santa Rosa, 2012.

\section{Objetivos específicos}

1. Evaluar los recursos y actividades didácticas que emplea el docente en el tema: Los Sistemas del Cuerpo Humano, de Ciencias, de sexto grado de la Escuela Santa Rosa.

2. Estudiar las principales actitudes y conductas referidas al proceso de aprendizaje, que posee los estudiantes del sexto grado de la Escuela Santa Rosa, en la asignatura de Ciencias, en el tema: Los Sistemas del Cuerpo Humano.

3. Diseñar un libro electrónico con el tema de Los Sistemas del Cuerpo Humano, en la asignatura de Ciencias para los alumnos de sexto grado de la Escuela Santa Rosa.

4. Identificar el impacto de la implementación del libro electrónico: Los Sistemas del Cuerpo Humano en los estudiantes del sexto grado de la Escuela Santa Rosa, en la asignatura de Ciencias.

\section{METODOLOGÍA}

\section{Tipo de estudio}

El tipo de investigación realizada respondió a un estudio descriptivo, de paradigma positivista y enfoque cuantitativo porque busca medir de manera objetiva un fenómeno que se da en el proceso de enseñanza y aprendizaje, el cual está relacionado con los recursos didácticos utilizados en la asignatura de Ciencias por los alumnos de sexto grado de la Escuela Santa Rosa y la utilización del libro electrónico como nuevo recurso.

\section{Participantes}

Para esta investigación se trabajó con el grupo de sexto grado, conformado por 18 estudiantes de la Escuela Santa Rosa y la docente a cargo del grupo. 


\section{Instrumentos}

Se empleó la observación con los alumnos de sexto grados en dos etapas, la primera para obtener información importante sobre el comportamiento y reacciones sobre los recursos que comúnmente utiliza la docente en la clase de Ciencias y la segunda etapa para conocer la reacción de los estudiantes al hacer uso del libro electrónico.

La entrevista fue aplicada a la docente a cargo del grupo de sexto grado con el fin de obtener información sobre dos aspectos, en primer lugar conocer cuáles son los recursos que comúnmente emplea en las clases de Ciencias, el por qué se emplean esos recursos, qué influencia tiene los recursos que emplea en relación con el rendimiento de los estudiantes y en segundo lugar para conocer la reacción del docente al emplearse el libro electrónico como recurso didáctico en la clase de Ciencias.

Se aplicaron 2 cuestionarios con preguntas estructuradas dirigidas a los estudiantes de sexto grado, el primer cuestionario contiene la debida presentación y explicación de la información solicitada, un bloque de preguntas, con dieciséis ítems relacionados con los tipos recursos que emplea la docente en las clases de Ciencias con el tema: Los Sistemas del Cuerpo Humano, la frecuencia con que los utiliza, la reacción y opinión de los alumnos por los recursos utilizados y el segundo cuestionario con once preguntas relacionadas con el uso del libro electrónico y la reacción de los estudiantes.

\section{Variables}

En el Cuadro 1 se detallan las variables utilizadas en esta investigación.

Cuadro 1. Descripción de variables para el desarrollo de la investigación

VARIABLES

Actividades que emplea la docente en el tema: Los Sistemas del Cuerpo Humano.

\section{DEFINICIÓN CONCEPTUAL}

Expresa una forma organizada y programada antes que el individuo la ejecute
DEFINICIÓN OPERACIONAL

Si el rango de las respuestas se encuentra entre $70 \%$ y $90 \%$ las actividades que emplea la docente son muy adecuadas.

Si el rango de las respuestas se encuentra entre $50 \%$ y $69 \%$ las actividades que emplea la docente son adecuadas.

Si el rango de las respuestas se encuentra entre $30 \%$ y $49 \%$ las actividades que emplea la docente son poco adecuadas.

$\mathrm{Si}$ el rango de las respuestas se encuentra entre $0 \%$ y $29 \%$ las actividades que emplea la docente no son nada adecuadas. 
Cuadro 1 (Continuación....). Descripción de variables para el desarrollo de la investigación

\begin{tabular}{|c|c|c|c|}
\hline VARIABLES & $\begin{array}{l}\text { DEFINICIÓN } \\
\text { CONCEPTUAL }\end{array}$ & DEFINICIÓN OPERACIONAL & $\begin{array}{l}\text { DEFINICIÓN } \\
\text { INSTRUMENTAL }\end{array}$ \\
\hline $\begin{array}{l}\text { Recursos didácticos } \\
\text { que emplea la do- } \\
\text { cente en el tema: Los } \\
\text { Sistemas del cuerpo } \\
\text { humano. }\end{array}$ & $\begin{array}{l}\text { Un recurso didáctico } \\
\text { es el material elabora- } \\
\text { do con la intención de } \\
\text { facilitar la función del } \\
\text { docente y el desarrollo } \\
\text { del alumno }\end{array}$ & $\begin{array}{l}\text { Si el rango de las respuestas se } \\
\text { encuentra entre } 70 \% \text { y } 90 \% \text { los } \\
\text { recursos que emplea la docente } \\
\text { no son adecuados. } \\
\text { Si el rango de las respuestas se } \\
\text { encuentra entre } 50 \% \text { y } 69 \% \text { los } \\
\text { recursos que emplea la docente } \\
\text { son poco adecuados. } \\
\text { Si el rango de las respuestas se } \\
\text { encuentra entre } 30 \% \text { y } 49 \% \text { los } \\
\text { recursos que emplea la docente } \\
\text { son adecuados. } \\
\text { Si el rango de las respuestas se } \\
\text { encuentra entre } 0 \% \text { y } 29 \% \text { los } \\
\text { recursos que emplea la docente } \\
\text { son muy adecuados. }\end{array}$ & $\begin{array}{l}\text { La variable se mide con las } \\
\text { respuestas de las preguntas } \\
\mathrm{N}^{\circ} 8 \text { y } \mathrm{N}^{\circ} 10 \text {, del cuestionario } \\
\mathrm{N}^{\circ} 1 \text { dirigido a estudiantes, } \\
\text { las respuestas de las pre- } \\
\text { guntas } \mathrm{N}^{\circ} 3 \text {, de la entrevista } \\
\text { dirigida a la docente y de las } \\
\text { anotaciones en la hoja de co- } \\
\text { tejo aplicada en un periodo } \\
\text { de seis o más lecciones en } \\
\text { diferentes situaciones. }\end{array}$ \\
\hline $\begin{array}{l}\text { Actitudes y conductas } \\
\text { referidas al proceso de } \\
\text { aprendizaje, que po- } \\
\text { seen los estudiantes } \\
\text { del sexto grado de la } \\
\text { Escuela Santa Rosa, en } \\
\text { la asignatura de Cien- } \\
\text { cias, en el tema: Los } \\
\text { Sistemas del cuerpo } \\
\text { humano. }\end{array}$ & $\begin{array}{l}\text { Se refiere a las con- } \\
\text { ductas y actitudes que } \\
\text { muestran los estu- } \\
\text { diantes en el proceso } \\
\text { de aprendizaje. }\end{array}$ & $\begin{array}{l}\text { Si el rango de las respuestas se } \\
\text { encuentra entre } 70 \% \text { y } 90 \% \text { las } \\
\text { actividades que emplea la do- } \\
\text { cente son muy adecuadas. } \\
\text { Si el rango de las respuestas se } \\
\text { encuentra entre } 50 \% \text { y } 69 \% \text { las } \\
\text { actividades que emplea la do- } \\
\text { cente son adecuadas. } \\
\text { Si el rango de las respuestas se } \\
\text { encuentra entre } 30 \% \text { y } 49 \% \text { las } \\
\text { actividades que emplea la do- } \\
\text { cente son poco adecuadas. } \\
\text { Si el rango de las respuestas se } \\
\text { encuentra entre o\% y } 29 \% \text { las } \\
\text { actividades que emplea la do- } \\
\text { cente no son nada adecuadas. }\end{array}$ & $\begin{array}{l}\text { La variable se mide con las } \\
\text { respuestas de las pregun- } \\
\text { tas } N^{\circ} 6, N^{\circ} 11 \text { y } N^{\circ} 12 \text { del } \\
\text { cuestionario } N^{\circ} 1 \text { dirigido a } \\
\text { estudiantes, las respuestas } \\
\text { de las preguntas } N^{\circ} 2 \text { y } N^{\circ} 11 \\
\text { de la entrevista dirigida a la } \\
\text { docente y de las anotaciones } \\
\text { en la hoja de cotejo aplicada } \\
\text { en un periodo de seis o más } \\
\text { lecciones en diferentes si- } \\
\text { tuaciones. }\end{array}$ \\
\hline
\end{tabular}


Cuadro 1 (Continuación....). Descripción de variables para el desarrollo de la investigación

\begin{tabular}{|c|c|c|c|}
\hline VARIABLES & $\begin{array}{l}\text { DEFINICIÓN } \\
\text { CONCEPTUAL }\end{array}$ & DEFINICIÓN OPERACIONAL & $\begin{array}{l}\text { DEFINICIÓN } \\
\text { INSTRUMENTAL }\end{array}$ \\
\hline $\begin{array}{l}\text { Impacto de la imple- } \\
\text { mentación del libro } \\
\text { electrónico: Los Siste- } \\
\text { mas del Cuerpo Huma- } \\
\text { no en los estudiantes } \\
\text { del sexto grado de la Es- } \\
\text { cuela Santa Rosa, en la } \\
\text { asignatura de Ciencias }\end{array}$ & $\begin{array}{l}\text { Se refiere a los resul- } \\
\text { tados reflejados en } \\
\text { los estudiantes al uti- } \\
\text { lizar una nueva herra- } \\
\text { mienta. }\end{array}$ & $\begin{array}{l}\text { Si el rango de las respuestas se } \\
\text { encuentra entre } 70 \% \text { y } 90 \% \text { las } \\
\text { actividades que emplea la do- } \\
\text { cente son muy adecuadas. } \\
\text { Si el rango de las respuestas se } \\
\text { encuentra entre } 50 \% \text { y } 69 \% \text { las } \\
\text { actividades que emplea la do- } \\
\text { cente son adecuadas. } \\
\text { Si el rango de las respuestas se } \\
\text { encuentra entre } 30 \% \text { y } 49 \% \text { las } \\
\text { actividades que emplea la do- } \\
\text { cente son poco adecuadas. } \\
\text { Si el rango de las respuestas se } \\
\text { encuentra entre o\% y } 29 \% \text { las } \\
\text { actividades que emplea la do- } \\
\text { cente no son nada adecuadas. }\end{array}$ & $\begin{array}{l}\text { La variable se mide con las } \\
\text { respuestas de las preguntas } \\
\mathrm{N}^{\circ} 2, \mathrm{~N}^{\circ} 3, \mathrm{~N}^{\circ} 5, \text { y } \mathrm{N}^{\circ} 6, \mathrm{~N}^{\circ} \\
7, \mathrm{~N}^{\circ} 8 \text { del cuestionario } \mathrm{N}^{\circ} 2 \\
\text { dirigido a estudiantes y las } \\
\text { respuestas de las preguntas } \\
\mathrm{N}^{\circ} 1, \mathrm{~N}^{\circ} 8 \text { de la entrevista } \mathrm{N}^{\circ} \\
2 \text { dirigida a la docente y de } \\
\text { las anotaciones en la hoja de } \\
\text { cotejo aplicada en un periodo } \\
\text { de seis o más lecciones en di- } \\
\text { ferentes situaciones. }\end{array}$ \\
\hline
\end{tabular}

\section{RESULTADOS}

En este apartado se describen e interpretan los datos obtenidos al aplicar los instrumentos con el propósito de analizar la información obteni$\mathrm{da}$, la cual se presenta en una serie de cuadros donde los ítems han sido agrupados por su similitud de acuerdo al objetivo propuesto y con su respetiva interpretación o comentario de cada uno de ellos.

\section{Actividades que emplea la docente en el tema: Los Sistemas del Cuerpo Humano}

El cuadro $\mathrm{N}^{\circ} 2$ muestra como de un total de 18 estudiantes de sexto grado, el $44,4 \%$ valora como regular las actividades en Ciencias que la docente les asigna, mientras que el 11,11\% indican que son malas y 5,5\% las caracterizan como muy malas. Esto refleja que la mayoría de los estudiantes opinan que las actividades que la docente les asigna no son de su total agrado.
La opinión que ofrezca el alumno de las actividades que la docente les asigna en clase es muy importante, dado que refleja qué tan interesantes, retadoras, y agradables son para ellos para lograr así promover y alcanzar un aprendizaje de gran calidad.

Cuadro 2. Valoración por parte de los estudiantes de las actividades que asigna la docente en la asignatura de Ciencias

\begin{tabular}{|l|c|c|}
\hline ASPECTOS & $\begin{array}{c}\text { CANTIDAD DE } \\
\text { ALUMNOS }\end{array}$ & $\%$ \\
\hline Muy buenas & 3 & 16,6 \\
\hline Buenas & 4 & 22,22 \\
\hline Regulares & 8 & 44,44 \\
\hline Malas & 2 & 11,11 \\
\hline Muy malas & 1 & 5,5 \\
\hline Total & 18 & 100 \\
\hline
\end{tabular}

Fuente: Elaboración propia con base en cuestionario aplicado a estudiantes de sexto grado. 
El Cuadro $N^{\circ} 3$ refleja que el $55,55 \%$ de los 18 estudiantes consultados afirma que participa algunas veces en la clase de Ciencias, esto refleja una escasa participación de más de la mitad de los estudiantes. La observación realizada en el aula, comprobó cómo efectivamente algunos alumnos no participan directamente en las lecciones. Se limitan a escuchar con poca disposición a la docente y a poner atención a otras cuestiones dentro y fuera del aula, solamente el $22,22 \%$ de los alumnos afirmaron participar siempre en las lecciones de Ciencias.

Cuadro 3. Frecuencia con que participa el estudiante en la clase de Ciencias

\begin{tabular}{|l|c|c|}
\hline \multicolumn{1}{|c|}{ ASPECTOS } & $\begin{array}{c}\text { CANTIDAD DE } \\
\text { ALUMNOS }\end{array}$ & $\%$ \\
\hline Siempre & 4 & 22,22 \\
\hline Casi siempre & 3 & 16,6 \\
\hline Algunas veces & 10 & 55,55 \\
\hline Nunca & 1 & 5,5 \\
\hline Total & 18 & 100 \\
\hline
\end{tabular}

Fuente: Elaboración propia con base en cuestionario aplicado a estudiantes de sexto grado.

\section{- Recursos didácticos que emplea la docente en el tema: Los Sistemas del Cuerpo Humano}

El Cuadro $\mathrm{N}^{\circ} 4$ muestra como un porcentaje alto de alumnos de sexto grado afirmaron que las fotocopias es el recurso más utilizado en las clases de Ciencias, punto de vista de ellos que se corroboró mediante la observación realizada, ya que se determinó que hay poca variedad de recursos didácticos en la clase. También se pudo observar como la docente empleaba las fotocopias de forma excesiva, de hecho, fue el principal recurso utilizado en la lección, tanto para enseñar como para evaluar el tema de estudio.
Cuadro 4. Recursos utilizados por la docente para enseñar el tema: Los Sistemas del Cuerpo Humano

\begin{tabular}{|l|c|c|}
\hline $\begin{array}{c}\text { TIPOS DE } \\
\text { RECURSOS }\end{array}$ & $\begin{array}{c}\text { CANTIDAD DE } \\
\text { ALUMNOS }\end{array}$ & $\%$ \\
\hline Libro impreso & 3 & 16,6 \\
\hline Fotocopias & 14 & 77,77 \\
\hline Pizarra & 1 & 5,5 \\
\hline Videos & 0 & 0 \\
\hline Computadora & 0 & 0 \\
\hline Otros & 0 & 0 \\
\hline Total & 18 & 100 \\
\hline
\end{tabular}

Fuente: Elaboración propia con base en cuestionario aplicado a estudiantes de sexto grado.

\section{- Regularidad con que el alumno utiliza las fotocopias del libro de Ciencias cuando se está trabajando el tema: Los Sistemas del Cuerpo Humano}

El 83,33\% de los estudiantes afirmaron utilizar siempre las fotocopias del libro de Ciencias, dicho porcentaje demuestra que los recursos utilizados no son nada adecuados, según Zurriaga y Hermoso (1991), el libro de texto es el material didáctico dominante y casi exclusivo de muchas escuelas que siguen una metodología tradicional, muchos docentes aceptan la información contenida en los libros de texto en su totalidad, es decir, sin revisarla, sin reinterpretarla e incluso, sin alterala, los libros de texto o las fotocopias son necesarios siempre y cuando no sean los únicos materiales. Por esto, en la actualidad las nuevas tecnologías que abarcan un conjunto muy variado de recursos, herramientas y equipos, pueden proporcionar apoyo a la enseñanza y el aprendizaje en una amplia variedad de formas, como lo es en este caso el libro electrónico. 
Reacción de los estudiantes sobre el uso constante de las fotocopias del libro de Ciencias cuando se está trabajando el tema: Los Sistemas del Cuerpo Humano

Como se observa en el Cuadro 5, se pudo corroborar que más de la mitad de los alumnos dicen que el uso constante de las fotocopias del libro de Ciencias es aburrido, cuestión sobre lo cual también se le preguntó a la docente, respecto a cuál era la reacción que mostraban los estudiantes cuando utilizan las fotocopias y sobre esto la docente contestó: "Algunos estudiantes muestran interés, pero otros no tanto, porque no les gusta leer" Bonilla, L. (comunicación personal, entrevista, 19 de abril, 2012).

Cuadro 5. Regularidad con que el alumno utiliza las fotocopias del libro de Ciencias cuando se está trabajando el tema: Los Sistemas del Cuerpo Humano

\begin{tabular}{|l|c|c|}
\hline FRECUENCIA & $\begin{array}{c}\text { CANTIDAD DE } \\
\text { ALUMNOS }\end{array}$ & $\%$ \\
\hline Siempre & 15 & 83,33 \\
\hline Casi siempre & 2 & 11,11 \\
\hline Algunas veces & 1 & 5,5 \\
\hline Nunca & 0 & 0 \\
\hline Total & 18 & 100 \\
\hline
\end{tabular}

Fuente: Elaboración propia con base en cuestionario aplicado a estudiantes de sexto grado.

Cuadro 6. Reacción de los estudiantes sobre el uso constante de las fotocopias del libro de Ciencias cuando se está trabajando el tema: Los Sistemas del Cuerpo Humano

\begin{tabular}{|l|c|c|}
\hline \multicolumn{1}{|c|}{ OPINIÓN } & $\begin{array}{c}\text { CANTIDAD DE } \\
\text { ALUMNOS }\end{array}$ & $\%$ \\
\hline Agradable & 3 & 16,66 \\
\hline Aburrido & 12 & 66,66 \\
\hline Cansado & 3 & 16,66 \\
\hline Total & 18 & 100 \\
\hline
\end{tabular}

Fuente: Elaboración propia con base en cuestionario aplicado a estudiantes de sexto grado.
Además, en la observación realizada, se pudo apreciar como la mayoría de los estudiantes mostraron una reacción negativa por tener que utilizar las fotocopias de forma constante.

\section{- Distribución de la opinión de los estudiantes si les gustaría que la docente les entregara recursos didácticos diferentes}

Casi todos los estudiantes opinaron que les gustaría utilizar recursos didácticos diferentes a los que habitualmente utilizan. Estamos con una generación de niños y jóvenes a los cuales les gusta todo en tecnología, las TIC ayudan cada día más en el proceso de enseñanza y aprendizaje generándose así un infinito número de posibilidades que dispone al docente para que el alumno consiga de una forma más sencilla y divertida los objetivos que se propone. Herramientas como el libro electrónico proporciona tanto al educador como al alumno una útil herramienta tecnológica posicionando al alumno como protagonista y actor de su propio aprendizaje. De tal forma, asistimos a una renovación didáctica en las aulas donde se pone en práctica una metodología activa e innovadora que motiva al alumnado en las diferentes disciplinas o materias en este caso en la asignatura de Ciencias.

Cuadro 7. Distribución de la opinión de los estudiantes si les gustaría que la docente les entregara recursos didácticos diferentes

\begin{tabular}{|l|c|c|}
\hline $\begin{array}{c}\text { OPINIÓN DEL } \\
\text { ALUMNO }\end{array}$ & $\begin{array}{c}\text { CANTIDAD DE } \\
\text { ALUMNOS }\end{array}$ & $\%$ \\
\hline $\mathrm{Si}$ & 17 & 94,44 \\
\hline No & 1 & 5,5 \\
\hline Total & 18 & 100 \\
\hline
\end{tabular}

Fuente: Elaboración propia con base en cuestionario aplicado a estudiantes de sexto grado. 
Valoración por parte de los estudiantes de las actividades que asigna la docente en la asignatura de Ciencias después de haber utilizado el libro electrónico

En el Cuadro $\mathrm{N}^{\circ} 8$ se puede apreciar como después de utilizar el libro electrónico un gran porcentaje de alumnos, el $72,22 \%$ consideran las actividades que la docente les asigna Como muy buenas, el impacto de la implementación del libro electrónico se considera muy adecuado, comparado con el dato obtenido cuando no existía el libro electrónico, y en donde solo el $16,6 \%$ de los mismos estudiantes consideraban las actividades que la docente le asignaba como muy buenas. Es, por lo tanto, notorio que la herramienta el libro electrónico ha mejorado y enriquecido las actividades que la docente asigna para enseñar el tema de los Sistema del Cuerpo Humano, pues el libro electrónico ha logrado contrarrestar el uso abusivo de la fotocopia y del libro impreso.

Cuadro 8. Valoración por parte de los estudiantes de las actividades que asigna la docente en la asignatura de Ciencias después de haber utilizado el libro electrónico

\begin{tabular}{|l|c|c|}
\hline ASPECTOS & $\begin{array}{c}\text { CANTIDAD DE } \\
\text { ALUMNOS }\end{array}$ & $\%$ \\
\hline Muy buenas & 13 & 72,22 \\
\hline Buenas & 4 & 22,22 \\
\hline Regulares & 1 & 5,5 \\
\hline Malas & 0 & 0 \\
\hline Muy Malas & 0 & 0 \\
\hline Total & 18 & 100 \\
\hline
\end{tabular}

Fuente: Elaboración propia con base en cuestionario aplicado a estudiantes de sexto grado.

\section{Frecuencia con que participa el estudiante en la clase de Ciencias con el libro electrónico}

Antes de hacer uso del libro electrónico se registró que sólo el $22,22 \%$ de los estudiantes participaba siempre en las clases de Ciencias, pero después de utilizar el libro electrónico el porcentaje de estudiantes que mencionaron que participaban siempre subió a $72,22 \%$, estos datos positivos se explican debido a que una de las características fundamentales del libro es la interactividad, misma que fomenta una actitud activa del alumno frente al carácter de exposición o pasivo que poseía al utilizar recursos tradicionales.

Cuadro 9. Frecuencia con que participa el estudiante en la clase de Ciencias cuando se estaba trabajando con el libro electrónico

\begin{tabular}{|l|c|c|}
\hline \multicolumn{1}{|c|}{ ASPECTOS } & $\begin{array}{c}\text { CANTIDAD DE } \\
\text { ALUMNOS }\end{array}$ & $\%$ \\
\hline Siempre & 13 & 72,22 \\
\hline Casi siempre & 4 & 22,22 \\
\hline Algunas veces & 1 & 5,5 \\
\hline Otros & 0 & 0 \\
\hline Total & 18 & 100 \\
\hline
\end{tabular}

Fuente: Elaboración propia con base en cuestionario aplicado a estudiantes de sexto grado.

\section{- Valoración de los estudiantes sobre cómo les pareció el libro electrónico: Los Sistemas del Cuerpo Humano utilizado en la clase de Ciencias}

Según Cuadro $N^{\circ} 10$, el libro electrónico obtuvo una valoración muy positiva por parte de los alumnos, ya que un $88,88 \%$ lo consideran interesante, según la definición operacional que se anotó en la variable $\mathrm{N}^{\circ} 4$ correspondiente a la pregunta $\mathrm{N}^{\circ} 6$ del cuestionario $\mathrm{N}^{\circ} 2$ aplicado 
a los estudiantes, indica que el impacto de la implementación del libro electrónico se considera muy adecuado, además en la observación realizada se comprobó que los estudiantes presentaron una reacción positiva al utilizar el libro electrónico y una mayor y mejor motivación durante todo este proceso educativo.

Este resultado es producto de que el libro rompe con el proceso rutinario de la clase en la cual se utilizaba de forma constante el libro físico, y más que el libro físico, la fotocopia en blanco y negro donde se pierde el asombro y el contenido científico. El libro electrónico demostró ser una herramienta innovadora, de fácil y agradable uso para los estudiantes. Tanto así que ha permitido enseñar y aprender el tema de los Sistemas del Cuerpo Humano de una forma agradable, logrando que los estudiantes no se vean sobrecargados con tanta información y de una forma poco atractiva como ocurría con el uso de las fotocopias del libro impreso.

Cuadro 10. Valoración de los estudiantes sobre cómo les pareció el libro electrónico: Los Sistemas del Cuerpo Humano utilizado en la clase de Ciencias

\begin{tabular}{|l|c|c|}
\hline VALORACIÓN & $\begin{array}{c}\text { CANTIDAD DE } \\
\text { ALUMNOS }\end{array}$ & $\%$ \\
\hline Interesante & 16 & 88,88 \\
\hline Agradable & 1 & 5,5 \\
\hline Retador & 0 & 0 \\
\hline Aburrido & 1 & 5,5 \\
\hline Desagradable & 0 & 0 \\
\hline Repetitivo & 0 & 0 \\
\hline Otros & 0 & 0 \\
\hline Total & 18 & 100 \\
\hline
\end{tabular}

Fuente: Elaboración propia con base en cuestionario aplicado a estudiantes de sexto grado.

\section{CONCLUSIONES}

» Se encontró que el único recurso utilizado por la docente en el área de Ciencias en el tema de los Sistemas del Cuerpo Humano es las fotocopias del libro de texto. Este abuso de fotocopias del libro de texto ha convertido la enseñanza de este tema en un proceso de memorización de amplios datos, que no favorecen la interacción entre los estudiantes.

»Las actividades que la docente diseña, el tipo de herramienta que se utiliza y la frecuencia con que las utiliza, afectan directamente el rendimiento, la actitud y conducta del estudiante en el proceso de enseñanza - aprendizaje.

» La dinámica de clase se vio favorecida al aplicar recursos poco tradicionales e innovadores como es el caso del libro electrónico, ya que generó atracción, interés y motivación en los estudiantes.

»Con el uso del libro electrónico se notó un cambio de actitud en la mayoría de los estudiantes ya que manifestaron agrado, curiosidad e interés por el tema visto en la clase de Ciencias.

\section{BIBLIOGRAFÍA}

Barbier, F. (2005). Historia del libro. Madrid, Alianza Editorial.

Delgado, C.E. (2009). Inclusión de las TIC en los programas educativos. San José, Costa Rica: Universidad Latina de Costa Rica.

Zurriaga, F. y Hermoso, T. (1999). La Pedagogía de Freinet. Alternativas al libro de Texto. Cuadernos de Pedagogía, nº 194, pág. 39-41. 
